we would have to invent something similar, as we need multiple "management packages" to implement global policies at less than global level.

The last book in this collection is the most substantive, perhaps because it is the only one by a single author. Weiss points out that the international legal order has been preoccupied with measures to take care of contemporary problems, yet our environmental challenges increasingly reach far into the future. Depletion of the ozone layer and global warming will entrain impoverishing repercussions for several generations to come, whereas mass extinction of species will leave a depauperate biosphere for perhaps a million years. How can the international community encompass the interests of future humankind on such a broad front?
Weiss examines the complexities of interdependence in this new temporal dimension, reviewing our understanding of rights, obligations, liability and "justice" as applied to nuclear waste, biological resources, water, soils and climate. A pioneering endeavour in a field of ultra-urgency, Weiss presents a firstclass statement of juridical intricacies, together with strategies for implementation such as codification, finance, management and incentives. From start to finish the book is informative and stimulating - hurrah, too, for the jargon-free style! The Brundtland commission would have found it an illuminating account of how to start to make our world one.

Norman Myers is a consultant in environment and development at Upper Meadow, Old Road, Oxford OX3 8SZ, UK.

\section{Times of change}

\section{John Bancroft}

Menopause: Evaluation, Treatment and Health Concerns. Edited by Charles B. Hammond, Florence P. Haseltine and Isaac Schiff. Liss: 1989. Pp.339. \$96, £76.

THE medical management of the menopause is an issue of growing interest and importance. As the evidence accumulates the complexity increases. From what was traditionally regarded as "the change", a normal developmental process sometimes with associated problems, we have now a situation where the long-term health consequences are being carefully weighed.

One school of thought advocates widespread use of hormone-replacement therapy to combat osteoporosis, a rapidly increasing cause of morbidity and mortality in elderly women. This school emphasizes the potential advantages of hormone-replacement therapy in reducing the risk of coronary artery disease in later life. Apart from its long term benefits, hormone-replacement therapy can provide obvious and sometimes dramatic relief from transitional symptoms of menopause such as hot flushes. Against such benefits are potential risks of cancer in the endometrium and breast. Such risks are difficult to evaluate, seem to be small and yet generate much concern.

A meeting on the menopause was held at the National Institutes of Health at Bethesda in May 1988. This volume reports the proceedings of that meeting, and is a useful, up-to-date overview. Its main emphasis is on the physical aspects, with good contributions on osteoporosis, cardiovascular implications and breast cancer. The intriguing evidence from animal studies for the effects of oestrogens in accelerating ovarian ageing is well reviewed, and some useful light is thrown on the unknown nature of vasomotor flushes.

One of the most controversial issues on menopause is its relation to mental health, in particular depression. The weight of evidence has suggested that whereas the transition through the menopause is not associated with any substantial increase in depressive illness, many women find themselves less able to cope with life's problems so that there is an overall modest increase in psychological morbidity. Two chapters report new data on this issue. The McKinleys carried out a longitudinal study in the United States of women passing through the menopause. These data are limited by the authors' reliance on telephone interviews, but the authors conclude that the menopause has no noticeable impact on mental health. Andrew Whitehead's British study further confirms the earlier picture of a modest increase in morbidity.

One issue which has not been addressed by either of these studies, or others before them, is the possibility that in a proportion of women falling levels of oestrogen after the menopause may contribute to depression whereas for others there may actually be an improvement in mental health and a reduced likelihood of depression, possibly because of their escape from the negative effects of the menstrual cycle. These two contrary effects of the menopause conform with clinical impressions. If they do exist, and are not studied separately, they could combine to produce a picture of no effect.

Those working in the field as well as those interested in struggling to assess the risks and benefits of hormone-replacement therapy will find this book a useful addition to the literature.

John Bancroft is at the MRC Reproductive Biology Unit, Chalmers Street, Edinburgh EH3 9EW, UK.

\section{Sticking to the facts}

\section{Martin J. Humphries}

Fibronectins. By Richard 0 . Hynes. Springer-Verlag: 1990. Pp. 546. £92, $\$ 129$.

MANY of the recent key advances in our understanding of the mechanisms of cellmatrix adhesion have come either directly or indirectly from work on the glycoprotein fibronectin. These include the amino-acid sequence data that gave insight into the modular nature of large extracellular proteins, the discovery of the RGD adhesive recognition signal peptide, the identification of alternatively spliced domains with functional significance and, most recently, identification of the integrin family of adhesion receptors. These, and related areas, have been reviewed over the past few years. But to place such topical areas of research interest in context, it is occasionally necessary to view them against the scaffold of accumulated knowledge from which they arose. The main strength of Fibronectins is that it provides a complete, up-to-date treatise on the cell biology, biochemistry and molecular biology of these fascinating molecules.

In his introduction to the book, Richard Hynes is reluctant to present chronological data on the number of publications on fibronectin, but notes that the rate is now sufficiently high to deter anyone from starting to compile a comprehensive review of the protein. The fact that he has achieved this goal is a testament to his scholarship. It is inevitable in any active research area that a review, never mind a book, is somewhat out of date before publication. However, to quote the preface, "the literature up to the end of 1988 is reviewed thoroughly and completely and major advances occurring up to the time of final copyediting (August 1989) are included". Nothing better could be expected.

The book is based broadly on the classical framework for a review; it has a brief, though always fascinating, historical perspective to the field, followed by a technically useful chapter on methodology, and then describes fibronectin distribution (in vivo and in vitro), its structure, its molecular interactions, and current views of its functions in embryonic development, oncogenic transformation, haemostasis, wound-healing, inflammation and microbial infection. Finally, there is a necessarily personal perspective of areas for future exploitation. I found the index a little lacking in detail, but at the same time had no real problem locating specific information. Perhaps someone less used to 